\title{
openheart Pacing therapy in the management of unexplained syncope: a tertiary care centre prospective study
}

\author{
Ekrem Yasa, ${ }^{1,2}$ Fabrizio Ricci, ${ }^{1,3}$ Hannes Holm, ${ }^{1,2}$ Torbjörn Persson, ${ }^{2}$ \\ Olle Melander, ${ }^{1,4}$ Richard Sutton, ${ }^{1,5}$ Viktor Hamrefors, ${ }^{1,4}$ Artur Fedorowski ${ }^{1,2}$
}

\begin{abstract}
- Additional material is published online only. To view please visit the journal online (http://dx.doi.org/10.1136/ openhrt-2019-001015).
\end{abstract}

To cite: Yasa E, Ricci F, Holm H, et al. Pacing therapy in the management of unexplained syncope: a tertiary care centre prospective study. Open Heart 2019;6:e001015. doi:10.1136/ openhrt-2019-001015

Received 18 January 2019 Revised 18 February 2019 Accepted 4 March 2019

(D) Check for updates

(C) Author(s) (or their employer(s)) 2019. Re-use permitted under CC BY-NC. No commercial re-use. See rights and permissions. Published by BMJ.

${ }^{1}$ Department of Clinical Sciences, Lund University, Malmö, Sweden

2Department of Cardiology, Skåne University Hospital, Malmö, Sweden

${ }^{3}$ Department of Neuroscience, Imaging and Clinical Sciences, Universita degli Studi Gabriele d'Annunzio Chieti e Pescara, Chieti Scalo, Italy

${ }^{4}$ Department of Internal Medicine, Skåne University Hospital, Malmö, Sweden ${ }^{5}$ National Heart and Lung Institute, Imperial College School of Medicine, London, United Kingdom

Correspondence to Dr Artur Fedorowski; artur. fedorowski@med.lu.se

\section{ABSTRACT}

Objective Pacemaker (PM) therapy is effective when syncope is associated with bradycardia, but syncope recurrences and fall injuries after PM implantation may occur. We aimed to survey indications and outcomes of PM implantation, following evaluation of unexplained syncope. Methods Among 1666 consecutive unpaced patients investigated in a tertiary syncope unit by carotidsinus massage (CSM), head-up tilt test (HUT) and ECG monitoring, 106 (6.4\%; age, $65 \pm 17$ years) received a PM. We assessed bradycardia detection methods, PM implantation indications, and explored incidence of recurrent syncope, fall-related fractures and mortality. Results Indications for PM therapy were met in 32/106 patients $(30 \%)$ by CSM, in $41 / 106(39 \%)$ by HUT, in $14 / 106$ patients (13\%) by implantable loop-recorder (ILR) and in 19/106 (18\%) by standard ECG. Sinus arrest with asystole was the predominant PM indication during CSM/ HUT and external ECG monitoring, whereas ILR detected proportionally the same numbers of asystole due to sinus arrest and atrioventricular block. During follow-up (median, 4.3 years), 15 patients (14\%) had syncope recurrence, 15 suffered fall-related fractures and 9 died. Neither syncope recurrence nor fall-related fractures were dependent on initial PM indication. The composite endpoint of recurrent syncope/fall-related fracture was associated with treated hypertension (OR 2.45; $95 \% \mathrm{Cl} 1.00$ to 6.0 ), reduced glomerular filtration rate (OR 1.63 per $10 \mathrm{~mL} / \mathrm{min} \downarrow ; 95 \% \mathrm{Cl}$ 1.22 to 2.19 ) and atrial fibrillation (OR 3.98; 95\% Cl 1.11 to 14.3). Recurrent syncope predicted increased mortality (OR 9.20; $95 \% \mathrm{Cl} 1.89$ to 44.8 ).

Conclusions Cardiovascular autonomic testing and ECG monitoring effectively identify pacing indications in patients with unexplained syncope. After PM implantation, treated hypertension, renal failure and atrial fibrillation predict syncope recurrence and fall-related injury. Recurrent syncope predicts increased mortality.

\section{INTRODUCTION}

Syncope is defined as transient loss of consciousness due to cerebral hypoperfusion, with a rapid onset, short duration and spontaneous complete recovery. ${ }^{12}$ The vast majority of syncopal events is caused by abnormal behaviour of the circulatory system, where three main mechanisms may be encountered:

\section{Key questions}

What is already known about this subject?

- Recurrent syncope and unexplained injuries in paced patients are important clinical problems commanding prompt assessment.

What does this study add?

- Indications for pacing in patients presenting with unexplained syncope can be identified by cardiovascular autonomic tests alone in over two-thirds of cases.

- Paced patients presenting with recurrent syncope and fall-related injuries often suffer from reflex syncope susceptibility and orthostatic hypotension.

- Treated hypertension, atrial fibrillation and renal dysfunction are independent predictors of syncope recurrence and fall-related injuries in pacemaker patients.

- Recurrent syncope in paced patients predicts higher mortality.

How might this impact on clinical practice?

- Recurrent syncope in paced patients flags progressive deterioration of cardiovascular and autonomic nervous systems and is associated with adverse outcome.

- Additional research is needed to understand the role of comorbidities and polypharmacy on the efficacy of pacing therapy for syncope and prevention of low-energy fractures.

reflex, autonomic failure or primary disease of the heart and great vessels. ${ }^{2}$ In the latter, bradycardia is the predominant mechanism. ${ }^{2}$

Cardiac pacing has been the established method of treating bradycardia since 1958 . Although very successful in cardiac syncope due to intrinsic atrioventricular block (AVB), with syncope recurrence rate of about $5 \%$ over 5 years, ${ }^{3}$ pacemaker (PM) therapy in reflex syncope of cardioinhibitory type (asystole $>3 \mathrm{~s}$ ) is not as effective. ${ }^{4}$ In the presence of hypotensive susceptibility indicated by a positive head-up tilt test (HUT), ${ }^{5}$ syncope recurrence rate may be as high as $25 \%-50 \%$, whereas negative HUT heralds pacing 
efficacy very similar to that in AVB. ${ }^{45}$ Thus, cardiac pacing is an effective treatment of syncope when applied in patients with either intrinsic AVB or in cardioinhibitory reflex syncope with a modest hypotensive susceptibility (so-called 'vasodepressor component'). This approach has been confirmed in the Syncope Unit Project-2, ${ }^{6} 7$ which combined a thorough autonomic assessment with long-term ECG monitoring. The current European Society of Cardiology (ESC) syncope guidelines state that pacing the reflex form is recommended in patients $>40$ years of age with recurrent attacks, absence of prodrome and traumatic falls (Class IIA). ${ }^{2}$ When syncope is unexplained, a stepwise algorithm has been proposed with cardiovascular autonomic assessment initially and prolonged ECG monitoring with implantable loop-recorder (ILR) to follow, if required. ${ }^{267}$

In this study, we explored the outcomes of the proposed strategy applied in a series of patients with unexplained syncope after initial evaluation. Further, we assessed the incidence of syncope recurrence and fall injury after PM implantation. Our study was performed in a tertiary referral centre with full access to all recommended diagnostic modalities and therapeutic options according to current syncope guidelines. ${ }^{12}$

\section{METHODS}

\section{Study setting and population}

The Syncope Study of Unselected Population in Malmö (SYSTEMA) project was initiated to investigate systematically and manage patients with unexplained syncope. ${ }^{8}$ Between August 2008 and December 2016, 1705 patients with suspected syncope that is, unexplained transient loss of consciousness by initial evaluation, who were referred to the tertiary Syncope Unit of Skåne University Hospital, Malmö, Sweden, were enrolled and underwent cardiovascular autonomic assessment including carotid sinus massage (CSM) and HUT. ${ }^{2}$ Following cardiovascular autonomic tests, patients were monitored using an ILR, if the aetiology of syncope could not be established. In addition to the main syncope workup, other tests may have been carried out, including exercise and external long-term ECG, echocardiography, coronary angiography, brain imaging and electroencephalogram, whenever appropriate.

\section{Examination protocol}

Cardiovascular autonomic tests included CSM, supine and upright, if appropriate, according to Newcastle protocol, ${ }^{9}$ and tilt-table testing at $60^{\circ}-70^{\circ}$ plus optional nitroglycerin provocation according to the Italian protocol. ${ }^{10}$ The patients were asked to take their regular medication and fast for 2 hours before the test, although they were allowed to drink water freely. Beat-to-beat blood pressure (BP) and ECG were continuously monitored using a non-invasive validated method (Nexfin monitor, BMEYE, Amsterdam, Netherlands), and subsequently analysed offline using a dedicated programme provided by the monitor manufacturer. In addition, the patients were asked to complete a questionnaire, which explored medical history, duration, frequency and features of syncope-related symptoms, smoking status, and current pharmacological treatment. The study complied with the Declaration of Helsinki, the Regional Ethical Review Board in Lund, Sweden accepted the study protocol (ref no $82 / 2008$ ), and all study participants gave their written informed consent.

\section{Diagnostic criteria of orthostatic hypotension, carotid sinus syndrome and reflex syncope}

The following diagnostic criteria were applied: (a) reproduction of symptoms (dizziness, lightheadedness, presyncope and syncope), if patients were able to recall conditions preceding syncope, and (b) conventional criteria of orthostatic hypotension $(\mathrm{OH})$, carotid sinus syndrome (CSS) and vasovagal reflex syncope (VVS). ${ }^{2}$ Briefly, $\mathrm{OH}$ was defined as sustained decrease in systolic BP (SBP) $\geq 20 \mathrm{~mm} \mathrm{Hg}$ and/or decrease in diastolic BP (DBP) $\geq 10 \mathrm{~mm} \mathrm{Hg}$ or SBP $<90 \mathrm{~mm} \mathrm{Hg}$, CSS as a fall in $\mathrm{SBP} \geq 50 \mathrm{~mm} \mathrm{Hg}$ and/or asystole $>3$ seconds with reproduction of syncope/symptoms while VVS as a reproduction of syncope associated with a characteristic pattern of pronounced hypotension with or without bradycardia/ asystole. ${ }^{2}$ The cardioinhibitory Vasovagal International Study (VASIS) IIB type of VVS was defined as asystole $>3$ seconds. ${ }^{11}$

\section{Post-test workup}

All patients were informed of test results, instructed how to cope with attacks, and complementary pharmacological and non-pharmacological interventions were applied according to current guidelines. ${ }^{2}$ Patients with asystolic cardioinhibitory reflex (VASIS IIB on CSS or VVS) and recurrent, traumatic or unexpected syncopal attacks, especially if aged $>40$ years, were offered pacing therapy without further ILR monitoring. For patients younger than 40 years, an individual risk assessment and open discussion with the patient preceded the decision to pace. If the tests results were inconclusive or syncope, diagnosis could not be established after first-line evaluation using autonomic tests, patients with little or no prodrome, recurrent and traumatic attacks received an ILR. ILR patients who had positive non-asystolic HUT and/or CSM were instructed how to counteract the hypotensive reflex tendency according to current guidelines. ${ }^{2}$ Those who showed asystole $>3 \mathrm{~s}$ during a symptomatic episode were offered PM therapy, regardless of HUT/CSM results. In selected cases, where the autonomic tests were negative or inconclusive, and resting ECG demonstrated bifascicular block or significant bradycardia $<40 \mathrm{bpm}$, patients were offered PM therapy based on their clinical characteristics such as advanced age, comorbidities and history of unpredictable syncope associated with serious trauma, as recommended by current guidelines. ${ }^{2}$ This decision was reached by consensus between syncope expert (AF) and PM implanting specialist (TP). In a subset of patients, if 
autonomic tests were performed during hospitalisation, and in-hospital external ECG monitoring (telemetry or Holter-monitoring) detected significant arrhythmia, PM was implanted without ILR monitoring.

The standard PM programming was dual chamber pacemaker (DDD) mode in a range 50-60 to 120-160/ min. In selected cases of HUT-induced asystolic reflex with strong vasodepressor component preceding asystole, $\mathrm{PM}$ was programmed in DDD mode with closed-loop stimulation (CLS) (Biotronik, Berlin, Germany), and in patients with permanent atrial fibrillation VVI mode was selected.

\section{Follow-up evaluation}

The first author (EY) reviewed the medical records of all patients with PM implantation retrieving the following data: date of PM implantation, PM indication, information on syncope recurrence or unprovoked fall injury associated with low-energy fracture, as a possible syncope-proxy, ${ }^{12}$ and date and cause of death during follow-up period through 31 December 2017 (median, 4.3 years; range 1.2-9.3 years). Data and aetiologies of syncope recurrences and fall-related traumatic injuries were obtained by reviewing the medical records of the events, including history, PM settings and memory, any additional tests performed (such as orthostatic tests) as well as the final diagnosis by the responsible physician. VVS and $\mathrm{OH}$ were judged as aetiological factors when they were diagnosed in accordance with guidelines ${ }^{12}$ and in case of discrepancy between the diagnosis originally suggested by the responsible physician and the senior author who reviewed the records, the diagnosis was changed accordingly. In case the diagnosis was uncertain, the endpoint was assessed by adjudication between the first (EY) and the senior author (AF). Following primary endpoints were considered in the analyses: first recurrent syncope, first fall-related low-energy fracture and composite endpoint of either recurrent syncope or low-energy fracture.

\section{Statistical analysis}

The main characteristics of the study population are presented as mean and SD for continuous variables, and percentages for categorical variables. Group differences in continuous variables were compared using analysis of variance, and dichotomous variables were compared using Pearson's $\chi^{2}$ test.

Logistic regression model was applied to assess the relationship between the composite primary endpoint (recurrent syncope or low-energy fracture) and clinical patient characteristics. Moreover, we analysed relations between post-PM implantation mortality, recurrent syncope and fall injuries. All tests were two-sided and $p$ value $<0.05$ was considered statistically significant. All calculations were performed using IBM SPSS Statistics software V.25.0 (SPSS, Chicago, IL, USA) and GraphPad Prism V.6.00, GraphPad Software (La Jolla, CA, USA), www.graphpad. com.
RESULTS

\section{Patient characteristics}

Of 1705 patients investigated, $39(2.3 \%)$ had a PM at the time of the evaluation and were excluded from the study. Of the remaining 1666 patients, 106 (6.4\%) received a new PM following evaluation (figure 1). Compared with the rest of the cohort, patients who received a PM following evaluation were older and more often men (table 1$)$. The majority of patients that received a new PM $(71 \%)$ were $>60$ years. Baseline characteristics stratified by age (over/under 60 years) are shown in online supplementary table S1.

\section{Pacing indications and outcomes in patients with new PMs}

The pacing indications and the diagnostic methods are reported in figure 2. In 73 of 106 patients $(68.9 \%)$, the pacing indications were identified during cardiovascular autonomic tests, that is, CSM/HUT, whereas a smaller proportion of pacing indications $(n=14)$ was found on ILR (13.2\%). Abnormal resting ECG constituted 7.5\%, whereas in-hospital ECG monitoring accounted for $10.4 \%$ of all PM indications.

Of all patients in the SYSTEMA cohort that were examined during the period of study, 32 of 215 patients with positive CSM (14.8\%) received PM while among 933 patients with positive HUT (ie, VVS), only 41 (4.4\%) were implanted with PM, of these seven with CLS-PM. There were three patients with asystolic vasovagal reflex and recurrent/traumatic falls who declined PM implantation (two women and one man, all $>40$ years). In the subset of patients investigated with ILR $(n=128)$, the 14 patients who received PM constituted a minority (10.9\%) of all monitored subjects.

Sinus arrest during CSM/HUT and external ECG monitoring was the predominant diagnosis leading to PM implantation. ILR detected proportionally the same numbers of sinus arrest without ventricular escape and AVB while intraventricular block dominated as indication obtained from resting ECG (table 2). Method of detection and pacing indications stratified according to age $<60 />60$ years are shown in (online supplementary tables S2, S3).

During follow-up (median 4.3 years; range 1.2-9.3 years), 15 patients $(14.2 \%)$ had syncope recurrence. The most common diagnoses in these patients were $\mathrm{OH}$ and VVS (table 3). Among patients in whom PM indications were detected by ILR, syncope reoccurred in 5 of $14(36 \%)$. Of these, PM indications were sinus arrest in two and AVB in three patients. In four of these five patients, cardiovascular autonomic tests performed prior to ILR monitoring and PM implantation demonstrated non-cardioinhibitory CSS $(n=2)$, vasovagal reflex without asystole $(n=1)$ and $\mathrm{OH}(\mathrm{n}=1)$, indicating a concurrent hypotensive susceptibility. Patients in whom PM indications were met during HUT had lower syncope recurrence rate; syncope reoccurred in $5 / 41$ patients (12.2 $\%)$. The corresponding PM indications were asystolic VVS ( $n=3)$ and VVS-induced AVB $(n=2)$, respectively. Of 


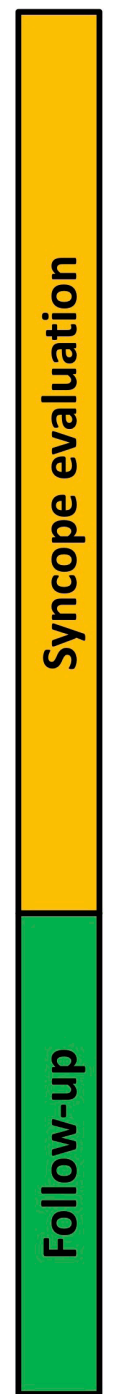

\section{Unexplained syncope or orthostatic intolerance $(n=1705)$}

PM in situ(n=39)

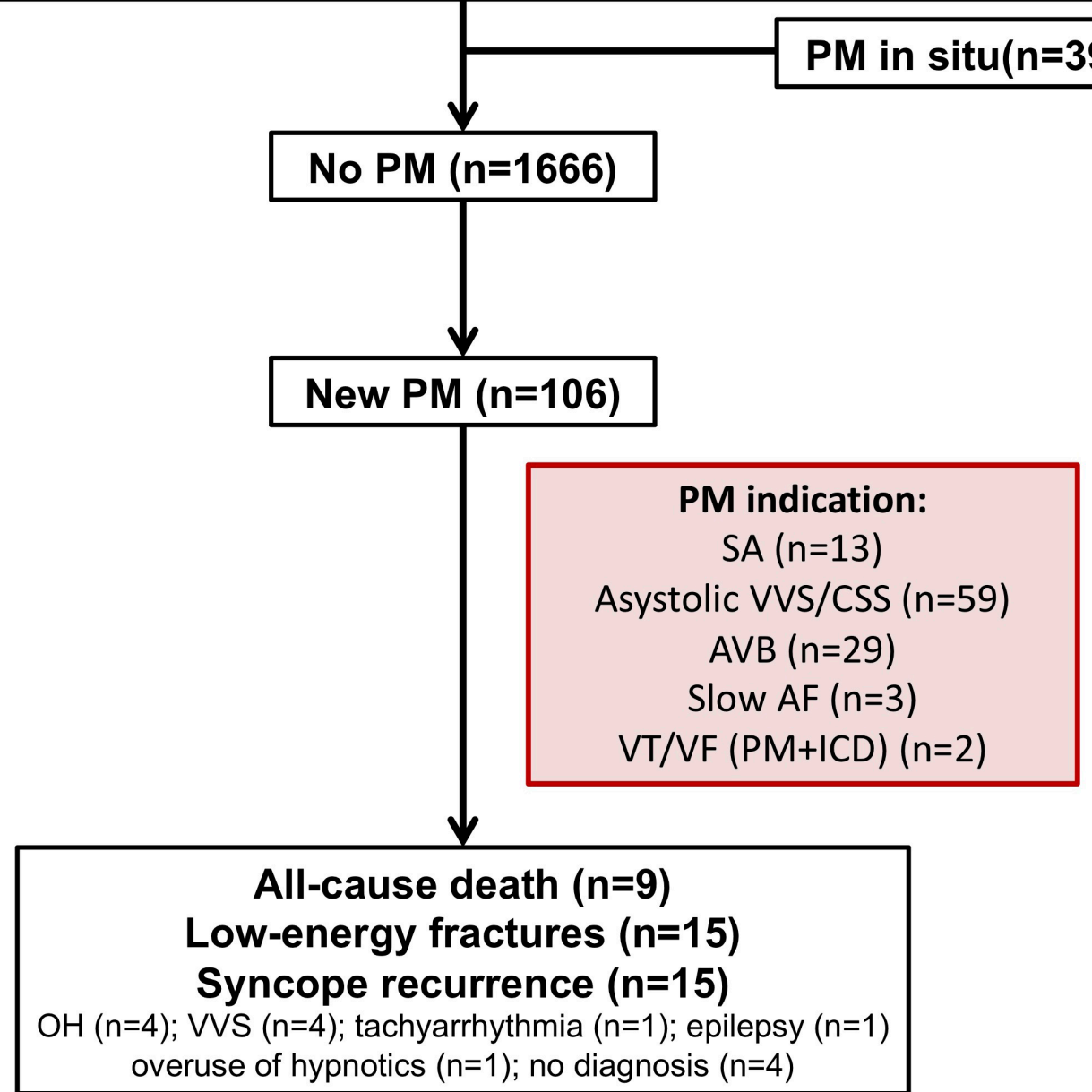

Figure 1 Flow chart of the study population. The diagram summarises the diagnostic workup and follow-up of patients presenting with unexplained syncope or symptoms of orthostatic intolerance. AF, atrial fibrillation; AVB, intraventricular/ atrioventricular block; CSS, carotid sinus syndrome; ICD, implantable cardioverter defibrillator; OH, orthostatic hypotension; PM, pacemaker; SA, sinus arrest; VT/VF, ventricular tachycardia/ventricular fibrillation; VVS, vasovagal syncope.

note, among seven patients who received CLS-PM, there were no syncope recurrences. Among 32 paced patients diagnosed with cardioinhibitory CSS there were five recurrences of syncope $(15.6 \%)$.

In all, 15 patients (14.2\%) suffered unexplained fall-related fractures during follow-up. Of these, six fractures occurred among 41 patients (15\%) in whom PM indication was found during HUT, 4 among 32 patients $(13 \%)$ diagnosed during CSM, 2 among 11 patients (18\%) diagnosed by ECG monitoring and 3 among 14 patients (21\%) diagnosed by ILR.

Using the composite endpoint, 28 (26\%) experienced either syncope or fall-related low-energy fracture during follow-up (15 syncope; 15 fractures, combined syncope/ fracture in two patients; figure 1). The clinical factors identified at the time of evaluation, associated with the endpoint, were hypertension and concurrent antihypertensive treatment with either thiazides or angiotensin receptor blockers, or both, reduced renal function and atrial fibrillation (table 4). Results by age over/under 60 years are shown in Tables $\mathrm{S} 4$.

During follow-up, nine patients with newly implanted PM died: five patients with and four patients without preceding syncope recurrence. None of those deaths were PM or bradycardia related. Syncope recurrence was associated with mortality (OR 9.20; 95\% CI 1.89 to 44.8; $\mathrm{p}=0.006$ ) after adjustment for age and sex. Furthermore, three patients with fall-related fracture died, and in the remaining group there were accordingly six deaths. Fall-related fractures were not associated with increased mortality (OR: 2.62; $95 \%$ CI, 0.52 to $13.3 ; \mathrm{p}=0.25$ ).

\section{DISCUSSION}

In this study, we observed that (i) indications for pacing in patients presenting with unexplained syncope can be identified in $70 \%$ by cardiovascular autonomic tests, that is, CSM, head-up tilt testing and in $13 \%$ by insertable 
Table 1 Patient characteristics $(n=1666)$ at the time of initial evaluation stratified according to pacemaker status after completed syncope workup. Patients with previous pacemaker were excluded

\begin{tabular}{|c|c|c|c|}
\hline & $\begin{array}{l}\text { Patients } \\
\text { with new } \\
\text { pacemaker } \\
(n=106)\end{array}$ & $\begin{array}{l}\text { Patients } \\
\text { without } \\
\text { pacemaker } \\
(\mathrm{n}=1560)\end{array}$ & $P$ value \\
\hline Age, years & $65.5(16.8)$ & $50.9(21.8)$ & $<0.001$ \\
\hline Sex, \% female & 45.3 & 61.8 & $<0.001$ \\
\hline \multicolumn{4}{|l|}{ Reported history of } \\
\hline Syncope, $\%$ & 98.1 & 91.0 & 0.014 \\
\hline Dizziness, n \% & 68.3 & 72.9 & NS \\
\hline $\begin{array}{l}\text { Number of syncope } \\
\text { episodes, md (range) }\end{array}$ & $7(0-100)$ & $4(0-1350$ & NS \\
\hline $\begin{array}{l}\text { Duration of symptoms, } \\
\text { years, md (range) }\end{array}$ & $7(0-70)$ & $3(0-77)$ & $<0.001$ \\
\hline SBP, mm Hg & $139.1(22.2)$ & $130.9(22.4)$ & $<0.001$ \\
\hline $\mathrm{DBP}, \mathrm{mm} \mathrm{Hg}$ & $71.4(10.5)$ & $71.6(10.2)$ & NS \\
\hline $\begin{array}{l}\text { Resting heart rate, } \\
\text { bpm }\end{array}$ & $66.3(11.6)$ & $70.5(12.6)$ & $<0.001$ \\
\hline eGFR, mL/min & $79.3(27.8)$ & $96.4(35.6)$ & $<0.001$ \\
\hline $\mathrm{EF}, \%$ & $54(3)$ & $54(3)$ & 0.418 \\
\hline Hypertension, \% & 39.4 & 27.8 & 0.011 \\
\hline $\begin{array}{l}\text { Antihypertensive } \\
\text { therapy, } \%\end{array}$ & 39.8 & 33.4 & 0.185 \\
\hline ACE inhibitors & 10.7 & 9.6 & 0.721 \\
\hline ARB & 16.5 & 9.2 & 0.014 \\
\hline Thiazides & 10.7 & 6.4 & 0.090 \\
\hline Beta blockers* & 14.6 & 18.5 & 0.322 \\
\hline $\mathrm{CAD}, \%$ & 7.8 & 6.3 & NS \\
\hline Atrial fibrillation, \% & 10.4 & 6.3 & NS \\
\hline Heart failure, \% & 6.8 & 3.1 & $<0.001$ \\
\hline
\end{tabular}

*Beta blockers were discontinued prior to the examination. ARB, angiotensin II receptor blockers; CAD, coronary artery disease; DBP, diastolic blood pressure; EF, ejection fraction; eGFR, estimated glomerular filtration rate;SBP, systolic blood pressure.

cardiac monitors; (ii) recurrent syncope and traumatic falls following PM implantation are common and over-represented in patients with hypertension taking antihypertensive therapy, atrial fibrillation and renal dysfunction and (iii) recurrent syncope after PM implantation is associated with increased mortality.

The literature on pacing has hitherto largely focused on ECG diagnosis in order to select patients for successful pacing, reaffirmed by the 2013 ESC guidelines on pacing. ${ }^{13}$ Follow-up of patients with clear ECG pacing indication has not been widely assessed, the emphasis being on technical faults and comorbidity, some induced by pacing, for example, heart failure. Recurrent syncope has had less attention than it deserved, being its relative rarity a partial explanation. Early series about recurrent syncope in PM recipients raised the possibility of autonomic causes, although a full battery of autonomic investigations was not available to those investigators. ${ }^{14}{ }^{15}$ Using a prospective investigational protocol including cardiovascular autonomic tests, CSM and HUT, completed, when necessary, by ILR, we have been able to provide insights into the aetiological and prognostic significance of syncope recurrence after PM implantation. In particular, we have shown that recurrence during follow-up is relatively common compared with the known data on AVB patients who are permanently paced. ${ }^{3}{ }^{16}$ Sinus arrest without ventricular escape was associated frequently with recurrent syncope aetiology in our patient group among those with recurrent syncope $(41 \%)$; thus, it should be considered that many of these patients have the 'extrinsic' form of sinus node disease ${ }^{15}$ implying that these patients also have reflex syncope. ${ }^{2}$

Our results affirm the importance of a comprehensive diagnostic workup before a decision is made to implant a PM in patients without a clear explanation for syncope, as this may influence selection of pacing as therapy, type of device to be implanted and its programming. Moreover, concentrating resources and expertise in a dedicated facility - that is, syncope unit ${ }^{17}$-might be another important factor to achieve optimal diagnostic and therapeutic efficacy of unexplained syncope management. Our results seem to support this approach as cardiovascular autonomic tests are not widely available and cardiologists may have limited knowledge of test interpretation.

Another prominent aspect of this study is association between syncope recurrences and prevalent hypertension. Patients who are hypertensive and receiving antihypertensive medication tend to form a substantial part of the paced patient population. From our study, it appears that hypertensive patients are particularly vulnerable to recurrent syncope, likely due to excessive antihypertensive therapy. This is in line with the results of the Systolic Blood Pressure Intervention Trial (SPRINT) and Action to Control Cardiovascular Risk in Diabetes Blood Pressure (ACCORD BP) trials, ${ }^{18} 19$ where serious adverse events defined as hypotension and syncope occurred more frequently in the intensive-treatment group. The possible unwanted effect of antihypertensive therapy could be also explained by the higher prevalence of hypotensive susceptibility in our study population, which may offer greater sensitivity to BP-reducing drugs. ${ }^{5}$ Interestingly, hypertension is a risk factor for rehospitalisation after hip-fracture surgery, in many cases due to traumatic fall as also is treatment with thiazides. ${ }^{20}$ It should be kept in mind that recent North American hypertension guidelines recommend even lower therapeutic goals (BP $<130 / 80 \mathrm{~mm} \mathrm{Hg}$ ), which should be considered reservedly when treating patients with history of syncope. ${ }^{21}$ Notably, the Stop vasodepressor drugs in reflex syncope (STOP-VD) trial $^{22}$ has shown that recurrence of syncope and presyncope could be significantly reduced by discontinuing/reducing vasoactive therapy in most elderly patients affected by reflex vasodepressor syncope. 


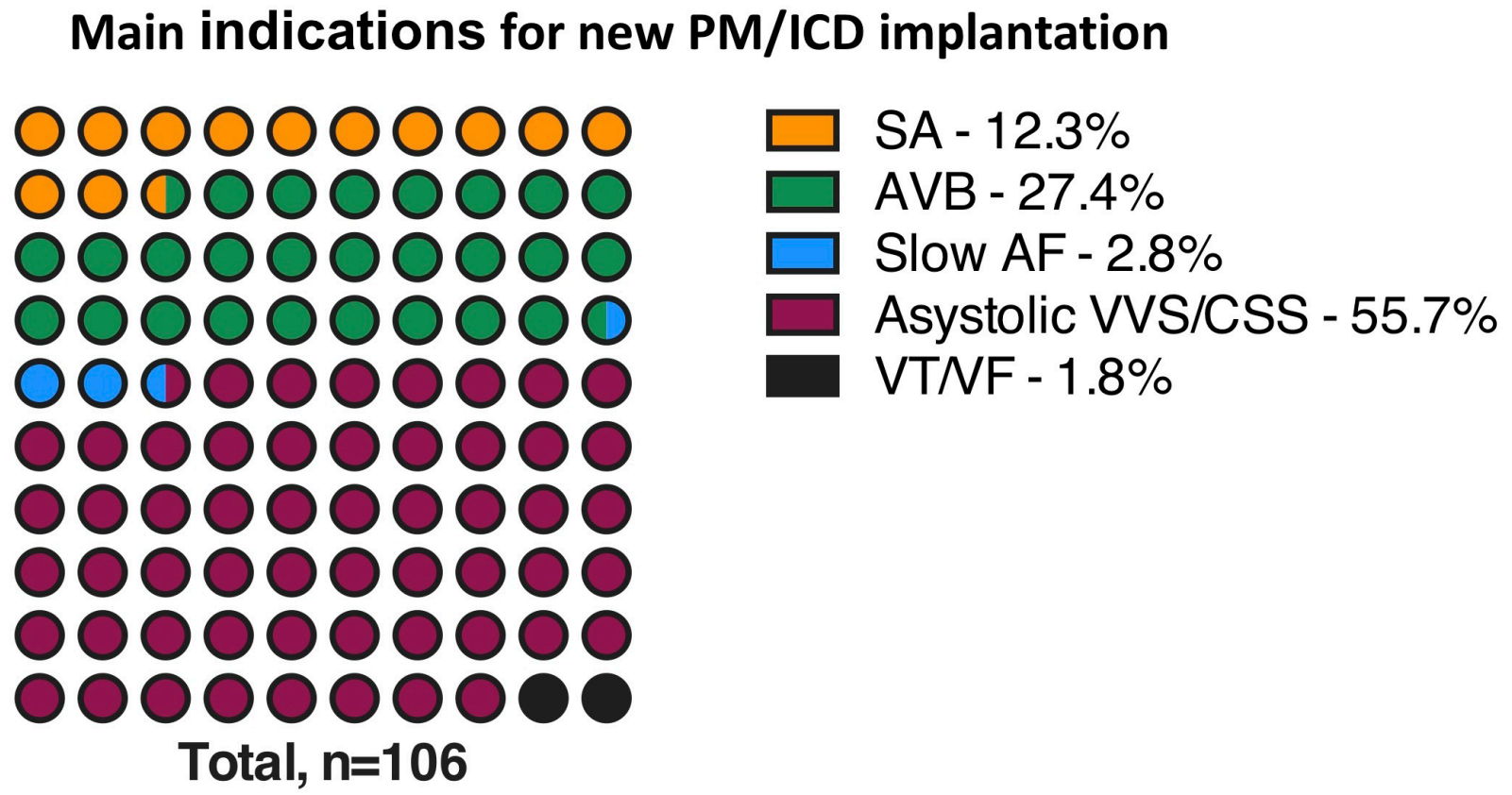

\section{Indications for new PM/ICD implantation by primary method of diagnosis}

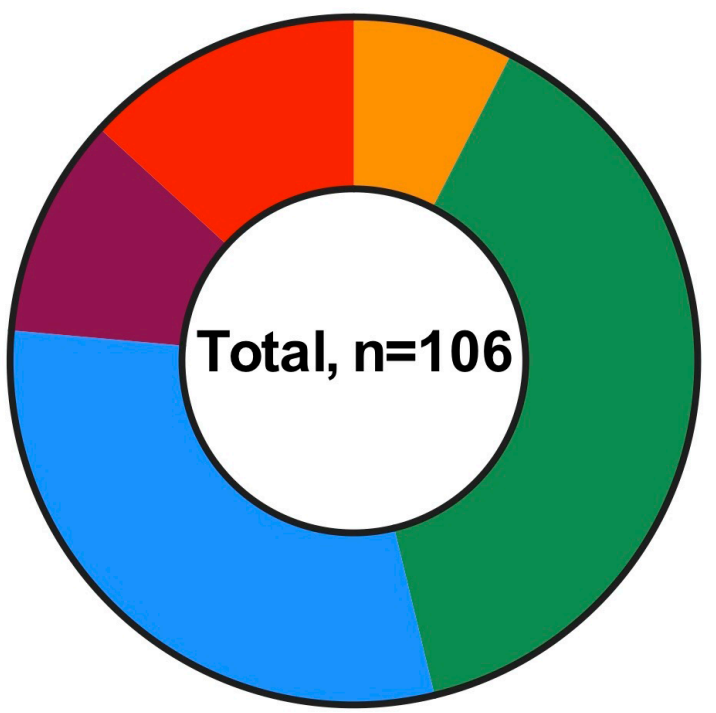

$\square$ Resting ECG - 7.5\%
$\square$ HUT - 38.7\%
$\square$ CSM - 30.2\%
$\square$ Holter ECG - $10.4 \%$
$\square$ ILR - $13.2 \%$

Figure 2 Main indications for new PM/ICD implantation and primary methods of diagnosis; 73 of the 106 patients (69 \%) revealed a pacing indication during HUT or CSM: asystolic VVS/CSS in 59 (81\%) cases, AVB in 13 (18\%) and slow AF in 1 (1\%). AF, atrial fibrillation; AVB, atrioventricular block; CSM, carotid-sinus massage; CSS, carotid sinus syndrome; HUT, headup tilt test; ICD, implantable cardioverter defibrillator; ILR, implantable loop-recorder; PM, pacemaker; SA, sinus arrest; VT/VF, ventricular tachycardia/ventricular fibrillation; VVS, vasovagal syncope.

In our cohort, two other factors were associated with syncope recurrence and fall-related fractures after PM implantation: renal failure and atrial fibrillation. This is also in agreement with previous findings from a Danish nation-wide study of patients with first-time syncope where atrial fibrillation and impaired renal function were found to be independent predictors of recurrent syncope, especially in the youngest segment of the population, that is, $<65$ years. ${ }^{23}{ }^{24}$ Similar findings were reported by an Irish group, where hypertension and AF were associated with increased fall propensity. ${ }^{25}$ Both conditions affect the compensatory mechanisms governed by autonomic and cardiovascular systems: renal failure hampers body fluid homeostasis, whereas atrial fibrillation portends a significant loss of autonomic control of chronotropic response, both crucial for baroreflex function. It is also plausible that hypertension is aetiologically associated with both reduced renal function and atrial fibrillation, leading to a vicious circle requiring careful judgement of risks and potential benefits of intensive BP reduction (figure 3 ).

While aggressive hypotensive therapy may serve to improve cardiovascular outcomes and other surrogate 
Table 2 Pacing indications according to the method of diagnosis in patients with newly implanted pacemaker after completed syncope workup

\begin{tabular}{|c|c|c|c|c|c|c|}
\hline & Resting ECG & HUT* $^{*}$ & CSM* $^{*}$ & External ECG monitoring & ILR & Total \\
\hline SA or asystolic reflex ${ }^{*} n$ & 1 & 33 & 26 & 6 & 6 & 72 \\
\hline Atrioventricular block, $\mathrm{n}$ & 7 & 8 & 5 & 3 & 6 & 29 \\
\hline Slow AF, n & 0 & 0 & 1 & 1 & 1 & 3 \\
\hline $\begin{array}{l}\text { SA plus } \\
\text { VT/VF, } n\end{array}$ & 0 & 0 & 0 & 1 & 1 & 2 \\
\hline Total & 8 & 41 & 32 & 11 & 14 & 106 \\
\hline
\end{tabular}

*In the cases where HUT or CSM were applied, the diagnosis was asystolic (cardioinhibitory) reflex and the absence of $p$-waves. AF, atrial fibrillation;CSM, carotid-sinus massage; HUT, head-up tilt test; ILR, implantable loop recorderSA, sinus arrest; VF, ventricular fibrillation; VT, ventricular tachycardia; VVS, vasovagal syncope.

endpoints of cardiovascular prognosis, there can be little doubt that syncopal recurrence has a significant impact on quality of life, remarkably debilitating in this age group, and is also associated with high healthcare costs, increased risk of fall-related injuries and cardiovascular and all-cause death. ${ }^{24}$ Particularly, hip fractures are major consequences of syncope-related falls and are associated with approximately $25 \%$ reduction of life expectancy and institutionalisation rates ranging between $8 \%$ and $34 \%$ in community-dwelling patients. ${ }^{26}$ Differentiation between falls and syncope is challenging, especially among elderly patients with cognitive impairment and experience from dedicated syncope and fall facilities reinforces the evidence of an overlap between these two entities, which are often indistinguishable and likely manifestation of the similar underlying pathophysiology. ${ }^{27}$ Both non-accidental falls and syncope show strong association with antihypertensive treatment and number of prevalent cardiovascular conditions including atrial fibrillation. ${ }^{27}$ A possible explanation for this overlap is that haemodynamic changes insufficient to cause critical cerebral hypoperfusion but sufficient to reduce cerebral perfusion play a part in falls and consequent low-energy fractures especially in older patients already compromised by

Table 3 The aetiology of syncope/T-LOC recurrence among patients who received pacemaker after completed syncope workup

\begin{tabular}{ll}
\hline & $\begin{array}{l}\text { All } \\
(\mathbf{n = 1 0 6 )}\end{array}$ \\
\hline No syncope recurrence, $\mathrm{n}(\%)$ & $91(85.8)$ \\
\hline Syncope recurrence, $\mathrm{n}(\%)$ & $15(14.2)$ \\
\hline Orthostatic hypotension, $\mathrm{n}(\%)$ & $4(26.7)$ \\
\hline Vasovagal syncope, $\mathrm{n}(\%)$ & $4(26.7)$ \\
\hline Tachyarrhythmia, $\mathrm{n}(\%)$ & $1(6.7)$ \\
\hline Epileptic seizure ${ }^{\star}, \mathrm{n}(\%)$ & $1(6.7)$ \\
\hline Hypnotics overuse ${ }^{\star}, \mathrm{n}(\%)$ & $1(6.7)$ \\
\hline No diagnosis, $\mathrm{n}(\%)$ & $4(26.7)$ \\
\hline
\end{tabular}

*Not syncope by definition.

T-LOC, transient loss of consciousness. gait and balance abnormalities and impaired protective reflexes. These results warrant further observational and interventional studies on the role of chronic conditions that may influence the efficacy of PM therapy in syncope.

Finally, although the number of patients who died during the follow-up period was relatively small, there was a distinct correlation between recurrent syncope and increased mortality, in concordance with our previous reports. ${ }^{28} 29$ Thus, clinicians should be vigilant when syncope recurs as it may indicate further deterioration of cardiovascular and autonomic systems or be a red flag signalling increased risk of falls, fractures, hospital admissions and other potentially life-threatening conditions.

\section{Strengths and limitations}

The principal strengths of this work were (i) the prospective nature of the study conducted in a tertiary referral syncope unit with full access to all recommended diagnostic modalities and therapeutic options according to current syncope guidelines and (ii) length of follow-up.

We acknowledge some limitations of the present work: (i) this is a single-centre observational study and our

Table 4 Factors associated with the composite endpoint of syncope recurrence and fall-related low-energy fracture $(n=28)$ among 106 patients who received pacemaker after completed syncope workup

\begin{tabular}{lll}
\hline & OR (95 \% Cl) & P value \\
\hline Age, per year & $1.03(1.00$ to 3.75$)$ & 0.081 \\
\hline Female sex & $1.57(0.66$ to 3.75$)$ & 0.306 \\
\hline Hypertension & $2.45(1.00$ to 6.00$)$ & 0.049 \\
Use of thiazides and/or ARB & $3.14(1.16$ to 8.49$)$ & 0.024 \\
\hline eGFR, per $10 \mathrm{~mL} /$ min decrease & $1.63(1.22$ to 2.19$)$ & 0.001 \\
\hline Atrial fibrillation & $3.98(1.11$ to 14.3$)$ & 0.034 \\
\hline Use of hypnotics & $2.96(0.40$ to 22.1$)$ & 0.290 \\
\hline Diagnosis of OH & $0.68(0.26$ to 1.73$)$ & 0.414 \\
\hline Diagnosis of VVS & $0.54(0.23$ to 1.30$)$ & 0.168 \\
\hline
\end{tabular}

ARBs, angiotensin receptor blocker $\mathrm{OH}$, orthostatic hypotension ; VVS, vasovagal syncope; eGFR, estimated glomerular filtration rate according to Cockcroft Gault formula. 


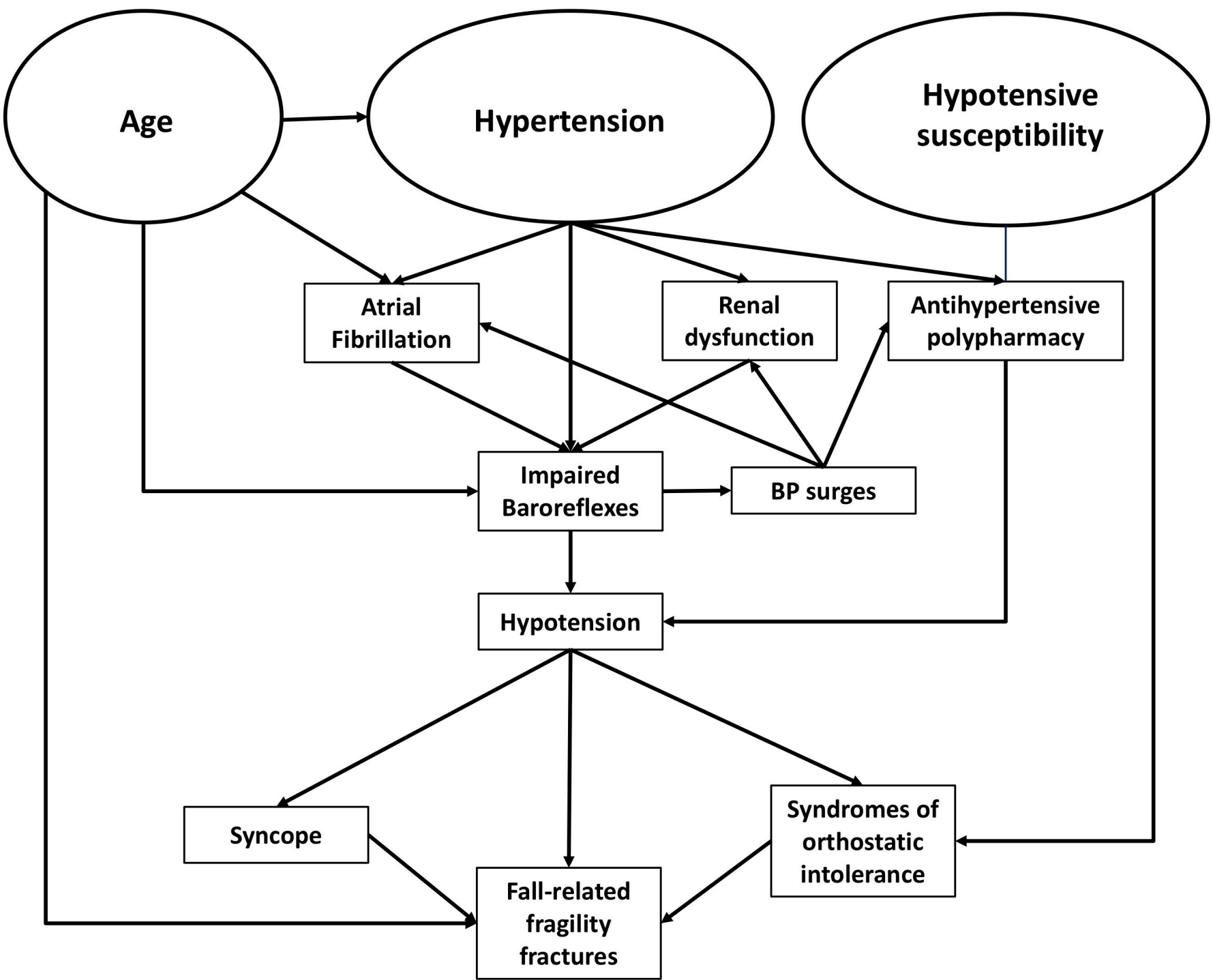

Figure 3 Pathophysiological mechanisms underlying impaired baroreflex function and recurrent syncope in paced patients.

results need confirmation in independent and larger samples; (ii) our study sample is small but in the light of our findings we felt that an early report is appropriate; (iii) our study sample reflects a selected population of individuals referred to a tertiary syncope unit and may not reflect the general syncope population and (iv) patients with PMs implanted due to primary cardiac arrhythmia detected prior to our evaluation in the syncope unit were not included.

\section{Conclusions}

Cardiovascular autonomic tests and insertable cardiac monitors reveal pacing indications in most patients presenting with unexplained syncope. In syncope patients with newly implanted PMs, prevalent hypertension associated with antihypertensive treatment, renal failure and atrial fibrillation may predict recurrent syncope and fall injury. Syncope recurrences in paced patients herald increased risk of death.

Contributors $\mathrm{FR}, \mathrm{AF}, \mathrm{HH}$ and $\mathrm{EY}$ had full access to all the data in the study and take responsibility of the data and accuracy of the data analysis. $\mathrm{OM}, \mathrm{AF}, \mathrm{HH}, \mathrm{VH}$ and TP contributed to the study conception and design. OM and AF contributed to the acquisition of data. All authors analysed and interpreted the data. AF was the study supervisor. AF, FR and VH did the statistical analysis. EY, FR, AF and VH drafted the manuscript with critical revision for important intellectual content from all authors.

Funding This work was supported by grants from the Swedish Heart and Lung Foundation, Malmö University Hospital, the Crafoord Foundation, the Ernhold Lundströms Research Foundation, the Region Skåne, and the Eva and Carl-Eric Larsson Foundation.

Competing interests AF reports personal fees from Cardiome Corp. and a patent Thermofisher pending outside the submitted work; RS reports personal fees and other from Medtronic Inc., Abbott Laboratories Inc. outside the submitted work; RS performs consultancy for Medtronic Inc.; RS is a member of the speaker's Bureau of Abbott Laboratories Inc.; RS is shareholder in Boston Scientific Inc., Edwards Lifesciences Inc., and Astrazeneca PLC; no other relationships oractivities that could appear to have influenced the submitted work.

Patient consent for publication Not required.

Provenance and peer review Not commissioned; externally peer reviewed.

Open access This is an open access article distributed in accordance with the Creative Commons Attribution Non Commercial (CC BY-NC 4.0) license, which permits others to distribute, remix, adapt, build upon this work non-commercially, and license their derivative works on different terms, provided the original work is properly cited, appropriate credit is given, any changes made indicated, and the use is non-commercial. See: http://creativecommons.org/licenses/by-nc/4.0/. 


\section{REFERENCES}

1. Shen WK, Sheldon RS, Benditt DG, et al. ACC/AHA/HRS guideline for the evaluation and management of patients with syncope: Executive summary: a report of the American College of Cardiology/American Heart Association Task Force on clinical practice guidelines and the heart rhythm Society. J Am Coll Cardiol 2017;2017:620-63.

2. Brignole M, Moya A, de Lange FJ, et al. ESC guidelines for the diagnosis and management of syncope. Eur Heart $J$ 2018;2018:1883-948.

3. Aste M, Oddone D, Donateo P, et al. Syncope in patients paced for atrioventricular block. Europace 2016;18:1735-9.

4. Sutton R. Should we treat severe vasovagal syncope with a pacemaker? J Intern Med 2017;281:554-61.

5. Sutton R, Brignole M. Twenty-eight years of research permit reinterpretation of tilt-testing: Hypotensive susceptibility rather than diagnosis. Eur Heart J 2014;35:2211-2.

6. Brignole M, Ammirati F, Arabia F, et al. Assessment of a standardized algorithm for cardiac pacing in older patients affected by severe unpredictable reflex syncopes. Eur Heart J 2015;36:1529-35.

7. Brignole M, Arabia F, Ammirati F, et al. Standardized algorithm for cardiac pacing in older patients affected by severe unpredictable reflex syncope: 3-year insights from the syncope unit project 2 (SUP 2) study. Europace 2016;18:1427-33.

8. Fedorowski A, Burri P, Juul-Möller S, et al. A dedicated investigation unit improves management of syncopal attacks (Syncope Study of Unselected Population in Malmo--SYSTEMA I). Europace 2010;12:1322-8

9. Parry SW, Reeve P, Lawson J, et al. The Newcastle protocols 2008: an update on head-up tilt table testing and the management of vasovagal syncope and related disorders. Heart 2009;95:416-20.

10. Bartoletti A, Alboni P, Ammirati F, et al. 'The Italian protocol': a simplified head-up tilt testing potentiated with oral nitroglycerin to assess patients with unexplained syncope. Europace 2000;2:339-42.

11. Brignole M, Menozzi C, Del Rosso A, et al. New classification of haemodynamics of vasovagal syncope: beyond the VASIS classification. Analysis of the pre-syncopal phase of the tilt test without and with nitroglycerin challenge. vasovagal syncope International study. Europace 2000;2:66-76.

12. Cronin H, Kenny RA. Cardiac causes for falls and their treatment. Clin Geriatr Med 2010;26:539-67.

13. Brignole M, Auricchio A, Baron-Esquivias G, et al. ESC guidelines on cardiac pacing and cardiac resynchronization therapy: the task Force on cardiac pacing and resynchronization therapy of the European Society of cardiology (ESC). developed in collaboration with the European heart rhythm Association (EHRA). Eur Heart $J$ 2013;2013:2281-329.

14. Pavlovic SU, Kocovic D, Djordjevic M, et al. The etiology of syncope in pacemaker patients. Pacing Clin Electrophysiol 1991;14:2086-91.
15. Sgarbossa EB, Pinski SL, Jaeger FJ, et al. Incidence and predictors of syncope in paced patients with Sick sinus syndrome. Pacing Clin Electrophysiol 1992;15:2055-60.

16. Langenfeld $\mathrm{H}$, Grimm W, Maisch $B$, et al. Course of symptoms and spontaneous ECG in pacemaker patients: a 5-year follow-up study. Pacing Clin Electrophysiol 1988;11:2198-206.

17. Kenny RA, Brignole M, Dan G-A, et al. Syncope Unit: rationale and requirement--the European Heart Rhythm Association position statement endorsed by the Heart Rhythm Society. Europace 2015;17:1325-40.

18. Cushman WC, Evans GW, Byington RP, et al. Effects of intensive blood-pressure control in type 2 diabetes mellitus. $N$ Engl $J$ Med 2010;362:1575-85.

19. Wright JT, Williamson JD, Whelton PK, et al. A randomized trial of intensive versus standard blood-pressure control. N Engl J Med 2015;373:2103-16.

20. Härstedt M, Rogmark C, Sutton R, et al. Polypharmacy and adverse outcomes after hip fracture surgery. J Orthop Surg Res 2016;11.

21. Whelton PK, Carey RM, Aronow WS, et al. ACC/AHA/AAPA $\mathrm{ABC} / \mathrm{ACPM} / \mathrm{AGS} / \mathrm{APh} / \mathrm{ASH} / \mathrm{ASPC} / \mathrm{NMA} / \mathrm{PCNA}$ guideline for the prevention, detection, evaluation, and management of high blood pressure in adults: Executive summary: a report of the American College of Cardiology/American Heart Association Task Force on clinical practice guidelines. Hypertension 2017;2018:1269-324.

22. Solari D, Tesi F, Unterhuber M, et al. Stop vasodepressor drugs in reflex syncope: a randomised controlled trial. Heart 2017;103:449-55.

23. Ruwald MH, Hansen ML, Lamberts $\mathrm{M}$, et al. Comparison of incidence, predictors, and the impact of co-morbidity and polypharmacy on the risk of recurrent syncope in patients $<85$ versus $\geq 85$ years of age. Am J Cardiol 2013;112:1610-5.

24. Ruwald MH, Numé A-K, Lamberts $M$, et al. Incidence and influence of hospitalization for recurrent syncope and its effect on short- and long-term all-cause and cardiovascular mortality. Am J Cardiol 2014;113:1744-50.

25. Jansen S, Bhangu J, de Rooij S, et al. The association of cardiovascular disorders and falls: a systematic review. J Am Med Dir Assoc 2016;17:193-9.

26. Braithwaite RS, Col NF, Wong JB. Estimating hip fracture morbidity, mortality and costs. J Am Geriatr Soc 2003;51:364-70.

27. Bhangu J, King-Kallimanis BL, Donoghue OA, et al. Falls, nonaccidental falls and syncope in community-dwelling adults aged 50 years and older: implications for cardiovascular assessment. PLoS One 2017;12:e0180997.

28. Yasa E, Ricci F, Magnusson M, et al. Cardiovascular risk after hospitalisation for unexplained syncope and orthostatic hypotension Heart 2018;104:487-93.

29. Ricci F, Sutton R, Palermi S, et al. Prognostic significance of noncardiac syncope in the general population: a systematic review and meta-analysis. J Cardiovasc Electrophysiol 2018;29:1641-7. 